arXiv:0907.4681[hep-th]

July 27, 2009.

V2: August 11, 2009

\title{
On superembedding approach to multiple D-brane system. D0 story.
}

\author{
Igor A. Bandos ${ }^{\dagger *}$ \\ † Department of Theoretical Physics, University of the Basque Country, \\ P.O. Box 644, 48080 Bilbao, Spain \\ and IKERBASQUE, the Basque Foundation for Science, 48011, Bilbao, Spain
}

We develop superembedding approach to multiple D-particle (D0-brane) system. In flat target $D=10$ type IIA superspace this produces the supersymmetric and Lorentz covariant version of the Matrix model equations. The equations following from our superembedding approach to multiple D0 in curved type IIA superspace shows the Myers 'dielectric brane effect', i.e. interaction with higher form gauge fields which do not interact with a single D0-brane.

\footnotetext{
* Also at A.I. Akhiezer Institute for Theoretical Physics, NSC Kharkov Institute of Physics \& Technology, UA 61108, Kharkov, Ukraine. E-mail: igor_bandos@ehu.es, bandos@ific.uv.es
} 


\section{Introduction}

Although the first appearance of D-branes is dated by late 80th [1, 2, 3, 4, their special rôle in String/M-theory was widely appreciated in middle 90 th when it was found that Dp-brane carries RR (Ramond-Ramond) charges i.e. interact with the antisymmetric tensor gauge fields $C_{p+1}, C_{p-1}$, ..., with respect to which the fundamental strings is neutral [5]. It was quickly understood that the low energy dynamics of multiple Dp-brane system is described by the maximal supersymmetric $d=p+1$ gauge theory with the gauge group $U(N)$ in the case of $\mathrm{N}$ D-branes [6]. Already this limit was quite productive [7] and, in particular, allowed the conjecture of $\mathrm{M}$ (atrix) theory that the Matrix model [8] considered as a theory of multiple D0-brane system, could provide a nonperturbative treatment of the M-theory.

The complete nonlinear supersymmetric action for a single $\mathrm{D} p$-brane was constructed in [9] for $p=2$ and in [10] for general $h^{2}$. It contains the nonlinear Dirac-Born-Infeld (DBI) term [6, 9, 11] and the Wess-Zumino (WZ) term describing the coupling to RR gauge fields [12]. Even before the general actions were constructed in [10], the superembedding approach [16] was developed for the case of Dp-branes in [13] where it was shown that that the supersymmetric equations of motion can be obtained in its frame starting from the basic superembedding equation (see below) 3 . For the discussion in this letter it will not be excessive to notice that similar story happened with M5-brane: its equations of motion had been derived in [17, in the frame of superembedding approach, before the covariant and supersymmetric action was constructed in [18].

As far as the nonlinear action for multiple D-brane systems is concerned, it was expected that this should be described by some non-Abelian generalization of the DBI plus WZ action. Tseytlin proposed to use the symmetric trace prescription to construct the non-Abelian DBI action for the case of purely bosonic spacetime filling D-brane [19, 11].

Although the search for a supersymmetric generalization of such non-Abelian DBI action has not been successful, in 1999 Myers used it as a starting point and applying a chain of dualities, derived the so-called 'dielectric brane action' [20] which is widely accepted for the description of multiple D-brane system. This action, however, does not possess neither supersymmetry nor Lorentz symmetry. In spite of a number of attempts, its Lorentz covariant and/or supersymmetric generalizations is not known in general, although some progress was reached for the cases of low dimensions $D$, low dimensional branes and low co-dimensional branes [21, 22, 23, 4 .

As far as the superembedding approach shown its efficiency in derivation of Dp-brane and M5brane equations, it looks natural to apply it in the search for equations of motion for the multiple $\mathrm{D} p$-brane system. In this letter we describe the results which this procedure gives for the simplest case of multiple D0-brane system.

We begin by a very brief review of superembedding approach to single $\mathrm{D} p$-branes for arbitrary $p$, with particular emphasis on D0-brane case, which provides a technical basis for our study. Then we argue in favor of the idea to search for the description of multiple $\mathrm{D} p$-brane systems by trying to define a possible nonlinear generalization of the non-Abelian SYM multiplet by some set of constraints on the $\mathrm{D} p$-brane worldvolume superspace $\mathcal{W}^{(p+1 \mid 16)}$. The embedding of this worldvolume superspace with $d=p+1$ bosonic and 16 fermionic directions into the type II target superspace $\Sigma^{(10 \mid 32)}$ is determined by the so-called superembedding equation and, in the case of higher branes ( $p \geq 6[32]$ ), also by constraints on the Abelian SYM gauge field characteristic of $\mathrm{D} p$-brane. We argue that the use of these constraints is useful also for a lower $p$ cases.

Then, turning to the case of multiple D0-brane, we propose the $\mathrm{d}=1 \mathcal{N}=16 \mathrm{SYM}$ constraints

\footnotetext{
${ }^{2}$ To be more precise, the actions of [9, 10] are complete modulo higher derivative corrections.

${ }^{3}$ The complete form of the $\mathrm{D} p$-brane equations of motion can be found in [14] and [15].

${ }^{4}$ Notice also very interesting minus one quantization approach using string with boundary fermions proposed in 24]. The name 'minus one quantization' is suggested by that, to reproduce Myers action in this scheme, one has to perform the quantization of these boundary fermion sector. We comment more on this approach in the concluding section.
} 
which express its field strength in terms of a nanoplet of $s u(N)$ valued superfields $\mathbb{X}^{i}$ obeying a superembedding-like equation $D_{\alpha} \mathbb{X}^{i}=4 i\left(\sigma^{0 i} \Psi\right)_{\alpha}$. The leading component of this superfield, appearing in the expression for the dimension 1 (spinor-spinor) field strength of the $S U(N)$ gauge superforms, $G_{\alpha \beta}=\sigma_{\alpha \beta}^{i} \mathbb{X}^{i}$, describe the relative motion of N D0-brane constituents of the system. We show that they produce a nonlinear equations of motion, which, in the case of flat target superspace, describe a non-Abelian $\mathrm{D}=10 \mathrm{SYM}$ dimensionally reduced to $d=1$ (the system which is used to define the Matrix model). However, the superembedding approach is also able to produce multiple D0-brane equations in an arbitrary type IIA superspace supergravity background (and it is not clear how to reproduce these equations just by SYM dimensional reduction). We analyze the general algebraic structure of the bosonic equations of motion for the multiple D0-brane in general type IIA supergravity background, as follows from superembedding approach, and show that these describe the Myers 'dielectric brane' effect of polarization of multiple $\mathrm{D} p$-brane system by external higher form fluxes, i.e. shows the coupling of multiple D0-brane system to the higher form gauge fields, which do not interact with a single D0-brane. We conclude by discussion on our results and interesting directions for future study.

1.1. Basic notation. Our notation are close to one in [25]. We denote the type II superspace coordinates by $Z \underline{M}=\left(x^{\mu}, \theta^{\underline{\alpha}}\right)(\mu=0,1, \ldots, 9, \underline{\underline{\alpha}}=1, \ldots, 32)$ and supervielbein form by

$$
E^{\underline{A}}:=d Z^{\underline{M}} E_{\underline{M}} \underline{A}(Z)=\left(E^{\underline{a}}, \mathcal{E}^{\underline{\alpha}}\right), \quad \mathcal{E}^{\underline{\alpha}}=\left\{\begin{array}{l}
\left(E^{\alpha 1}, E_{\alpha}^{2}\right) \text { for type IIA } \\
\left(E^{\alpha 1}, E^{\alpha 2}\right) \text { for type IIB }
\end{array}\right.
$$

$(\underline{a}=0,1, \ldots, 9, \underline{\alpha}=1, \ldots, 32)$. These are restricted by the set of supergravity constraints the most essential of which are collected in the expression for the bosonic torsion two form. For type IIA case these are

$$
T^{\underline{a}}:=D E^{\underline{a}}=-i\left(E^{1} \wedge \sigma^{\underline{a}} E^{1}+E^{2} \wedge \tilde{\sigma}^{\underline{a}} E^{2}\right),
$$

while the constraints for type IIB superspace is obtained from (1.2) by omiting tilde (replacing $\tilde{\sigma}^{\underline{a}}$ by $\tilde{\sigma}^{\underline{a}}$ in the second term in the brackets, $T^{\underline{a}}:=D E^{\underline{a}}=-i\left(E^{1} \wedge \sigma^{\underline{a}} E^{1}+E^{2} \wedge \sigma^{\underline{a}} E^{2}\right)$. Here $\sigma^{\underline{a}}:=\sigma_{\alpha \beta}^{\underline{a}}=\sigma_{\beta \alpha}^{\underline{a}}$ and $\tilde{\sigma}_{\underline{a}}:=\tilde{\sigma}_{\underline{a}}^{\alpha \beta}=\tilde{\sigma}_{\underline{a}}^{\beta \alpha}$, are $D=10$ Pauli matrices which obey $\sigma^{\underline{a}} \tilde{\sigma}^{\underline{b}}+\sigma^{\underline{b}} \tilde{\sigma}^{\underline{a}}=2 \eta^{\underline{a b}}$.

\section{Superembedding approach to a $\mathrm{D} p$-brane.}

\subsection{Worldvolume superspace $W^{(p+1 \mid 16)}$}

Following the so-called doubly supersymmetric twistor-like approach to superparticles and superstrings [26, 27, 28 $]^{5}$ the superembedding approach [16, 13, 17, 14, 29, 25] describes the dynamics of super-p-brane in terms of embedding of a worldvolume superspace into the target superspace. In the case of $\mathrm{D}=10 \mathrm{D} p$-branes (Dirichlet super-p-branes) the worldvolume superspace $\mathcal{W}^{(p+1 \mid 16)}$ has $d=p+1$ bosonic and 16 fermionic dimensions. We denote the local coordinates of $\mathcal{W}^{(p+1 \mid 16)}$ by $\zeta^{\mathcal{M}}=\left(\xi^{m}, \eta^{\check{\alpha}}\right)(m=0,1, . ., p, \check{\alpha}=1, \ldots, 16)$ the embedding of $\mathcal{W}^{(p+1 \mid 16)}$ into the $D=10$ type II target superspace $\Sigma^{(10 \mid 32)}$ can be described in terms of coordinate functions $\hat{Z}^{\underline{M}}(\zeta)=\left(\hat{x}^{\underline{m}}(\zeta), \hat{\theta}^{\underline{\alpha}}(\zeta)\right)$,

$$
W^{(p+1 \mid 16)} \in \Sigma^{(10 \mid 32)}: \quad Z^{\underline{M}}=\hat{Z}^{\underline{M}}(\zeta)=\left(\hat{x}^{\underline{\underline{m}}}(\zeta), \hat{\theta}^{\underline{\underline{\alpha}}}(\zeta)\right) .
$$

\subsection{The superembedding equation}

A particular beauty of the superembedding approach is that, for all known super- $p$-branes, the embedding of the worldvolume superspace into the target superspace is characterized by a universal

${ }^{5}$ See [29] for the review and more references. 
equation which is called the superembedding equation. This geometrical equation (the name 'geometrodynamic equation' was used in [28]) restricts the coordinate functions $\hat{Z} \underline{M}(\zeta)$ and, in some cases, completely determines the dynamics of superbrane.

To write the most general form of this superembedding equation let us denote the supervielbein of $W^{(p+1 \mid 16)}$ by

$$
e^{A}=d \zeta^{\mathcal{M}} e_{\mathcal{M}}^{A}(\zeta)=\left(e^{a}, e^{\alpha}\right), \quad a=0,1, \ldots, p, \quad \alpha=1, \ldots, 16,
$$

and write the general decomposition of the pull-back of the supervielbein of target type II superspace, $\hat{E}^{\underline{A}}:=E^{\underline{A}}(\hat{Z})$ on this basis,

$$
\hat{E}^{\underline{A}}:=E^{\underline{A}}(\hat{Z})=d \hat{Z}^{\underline{M}} E_{\underline{M}}^{\underline{A}}(\hat{Z})=e^{b} \hat{E}_{b}^{\underline{A}}+e^{\alpha} \hat{E}_{\alpha} \underline{A} .
$$

The superembedding equation states that the bosonic supervielbein form has zero projection on the worldvolume fermionic supervielbein form. This is to say, it reads

$$
\hat{E}_{\alpha} \underline{a}:=\nabla_{\alpha} \hat{Z}^{\underline{M}} E_{\underline{M}} \underline{\underline{a}}(\hat{Z})=0, \quad \nabla_{\alpha}:=e_{\alpha}^{\mathcal{M}}(\zeta) \partial_{\mathcal{M}}, \quad \zeta^{\mathcal{M}}=\left(\xi^{m}, \eta^{\check{\alpha}}\right) .
$$

It can be also presented in an equivalent form of

$$
\hat{E}^{i}:=\hat{E}^{\underline{a}} u_{\underline{a}}^{i}=0
$$

where $u_{\underline{a}}^{i}=u_{\underline{a}}^{i}(\zeta)$ are $(9-p)$ spacelike, mutually orthogonal and normalized 10-vector fields,

$$
u_{\underline{a}}^{i} u^{\underline{a} j}=-\delta^{i j} .
$$

Eq. (2.5) means that they are orthogonal to the worldvolume superspace. We can complete thier set till moving frame by adding $d=(p+1)$ mutually orthogonal and normalized $D$-vector fields $u_{\underline{a}}^{b}=u_{\underline{a}}^{b}(\zeta)$ which are tangential to the worldvolume superspace,

$$
u_{\underline{a}}^{a} u^{\underline{a}} i=0, \quad u_{\underline{a}}^{a} \eta^{\underline{a} b} u_{\underline{b}}^{b}=\eta^{a b}, \quad a, b=0,1, \ldots, p, \quad \underline{a}, \underline{b}=0,1, \ldots, 9 .
$$

The statement that $u_{\underline{a}}{ }^{b}$ vectors are tangential to the worldvolume superspace implies that their contraction with the pull-back $\hat{E}^{\underline{a}}$ of the target superspace bosonic supervielbein $E^{\underline{a}}$ provides us with a set of $d=(p+1)$ linearly independent nonvanishing one-forms, which can be used as bosonic supervielbein of the worldvolume superspace,

$$
\hat{E}^{a}:=\hat{E}^{\underline{b}} u_{\underline{b}}^{a}=e^{a} .
$$

This $e^{a}$ is referred to as induced by the (super)embedding. Eqs. (2.8) and (2.5) implies

$$
\hat{E}^{\underline{a}}=e^{b} u_{b}^{\underline{a}} .
$$

This is one more equivalent form of the superembedding equation.

The fermionic supervielbein form $e^{\alpha}$ of the worldvolume superspace $W^{(p+1 \mid 16)}$ can also be induced by superembedding. When describing Dp-branes, it is convenient to identify $e^{\alpha}$ with the pull-back to $W^{(p+1 \mid 16)}$ of, say, the first of two target space fermionic supervielbein forms

$$
e^{\alpha}=\hat{E}^{\alpha 1}
$$

Then the general decomposition of the second fermionic supervielbein form reads

$$
\left\{\begin{array}{ll}
\hat{E}_{\alpha}^{2}=e^{\beta} h_{\beta \alpha}+e^{a} \chi_{a \alpha} & \text { for IIA case } \\
\hat{E}^{\alpha 2}=e^{\beta} h_{\beta}{ }^{\alpha}+e^{a} \chi_{a}^{\alpha} & \text { for IIB case }
\end{array} .\right.
$$


As far as the induced spin connection and normal bundle connections are concerned, it is convenient to write the definition of the connections using the $S O(1,9) \times S O(1, p) \times S O(9-p)$ covariant derivative action on the moving frame vector, [16],

$$
D \underline{u}_{\underline{b}}^{a}=u_{\underline{b}}^{i} \Omega^{a i}, \quad D u_{\underline{b}}^{i}=u_{\underline{b} a} \Omega^{a i}
$$

Both equations in (2.12) involve the covariant 1-form $\Omega^{a i}$ which describes extrinsic geometry of $W^{(p+1 \mid 16)}$ embedded into the type IIB superspace and provides the supersymmetric (and superform) generalization of the so-called second fundamental form of the classical surface theory (see [16] for more discussion).

The selfconsistency condition for the superembedding equation Eq. (2.5) gives, in particular, an algebraic equation for the spin tensor $h$ in (2.11). For type IIA it reads

$$
h \tilde{\sigma}^{\underline{b}} h^{T} u_{\underline{b}}^{i}=-\sigma^{\underline{b}} u_{\underline{b}}^{i} .
$$

while for type IIB it is given by $h \sigma^{\underline{b}} h^{T} u_{\underline{\underline{b}}}^{i}=-\sigma^{\underline{b}} u_{\underline{\underline{b}}}^{i}$ (again $\tilde{\sigma}^{\underline{b}} \mapsto \sigma^{\underline{b}}$ rule).

\subsection{Constraints for the worldvolume gauge field.}

The constraints for the worldvolume gauge field strength of the $D p$-brane have the universal form

$$
F_{2}:=d A-\hat{B}_{2}=\frac{1}{2} e^{b} \wedge e^{a} F_{a b},
$$

where $\hat{B}_{2}$ is the pull-back to the worldvolume superspace $W^{(p+1 \mid 16)}$ of the type IIB NS-NS superform potential $B_{2}$. The field strength of this is restricted by the constraints which, for type IIA case, can be collected in the following differential form expression

$$
H_{3}:=d B_{2}=-i E^{\underline{a}} \wedge\left(E^{1} \wedge \sigma_{\underline{a}} E^{1}-E^{2} \wedge \tilde{\sigma}_{\underline{a}} E^{2}\right)+\frac{1}{3 !} E^{\underline{c}_{3}} \wedge E^{\underline{c}_{2}} \wedge E^{\underline{c}_{1}} H_{\underline{c}_{1} \underline{c}_{2} \underline{c}_{3}} .
$$

The lowest dimensional ( $\left.\operatorname{dim} 2, \propto e^{\gamma} \wedge e^{\beta} \wedge e^{a}\right)$ component of the Bianchi identities

$$
d F_{2}=-\hat{H}_{3}
$$

implies

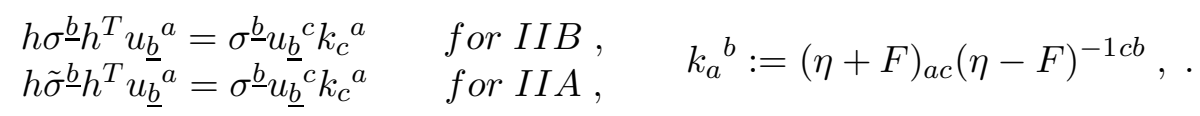

Notice that this equation relates the spin-tensor $h$, appearing in the decomposition of the pull-back of fermionic vielbein, and the gauge field strength $F_{a b}$. One can easily check that the matrix $k$ constrcuted from $F_{a b}$ as in (2.17) is $\mathrm{SO}(1, \mathrm{p})$ group valued, i.e. it obeys $k \eta k^{T}=\eta$ [31, 15],

$$
k=(\eta+F)(\eta-F)^{-1} \quad \in \quad S O(1,9) .
$$

Further study shows that the system of superembedding equation plus the worldvolume gauge field constraints (2.14) always contain the dynamical equations among their consequences (and for $p \leq 6 \mathrm{D} p$-branes [32] the superembedding equation along suffice for this purposes). However, the details of derivation are $p$-dependent so that we turn now to the case of D0-brane which is of our main interest here. 6

\footnotetext{
${ }^{6}$ This is the place to note that the off-shell worldline superfield formulations of massive $N=2$ superparticles in $D=2,3$ and 4 , which are the lower-dimensional (and lower supersymmetric) counterparts of a single D0-brane, were first considered in [27].
} 


\section{D0-brane in superembedding approach}

In the case of D0-brane there are nine spacelike directions orthogonal to worldline and the tangent to the worldline gives a time-like directions, so that the corresponding moving frame vectors $\left(u_{\underline{a}}^{0}, u_{\underline{a}}^{i}\right)$ obey

$$
u_{\underline{a}}^{0} u^{\underline{a} 0}=1, \quad u_{\underline{a}}^{i} u^{\underline{a} 0}=0, \quad u_{\underline{a}}^{i} u^{\underline{a} j}=-\delta^{i j} .
$$

The worldvolume superspace $W^{(1 \mid 16)}$ has only one bosonic direction, $e^{a} \mapsto e^{0}$ and the superembedding equation can be written as (see (2.9))

$$
\hat{E}^{\underline{a}}=e^{0} u_{0}^{\underline{a}},
$$

while the fermionic supervielbein forms read (see (2.10)

$$
\hat{E}^{\alpha 1}=e^{\alpha}, \quad \hat{E}_{\alpha}^{2}=e^{\beta} h_{\beta \alpha}+e^{0} \chi_{\alpha} .
$$

It is convenient to write the selfconsistency conditions for the superembedding equation (3.20) in the form of

$$
h \tilde{\sigma}^{i} h^{T}=-\sigma^{i} .
$$

using the simplified notation

$$
\sigma_{\alpha \beta}^{0}:=\sigma_{\alpha \beta}^{\underline{b}} \underline{u}^{0}, \quad \sigma_{\alpha \beta}^{i}:=\sigma_{\alpha \beta}^{\underline{b}} \underline{u}^{i} .
$$

These are suggestive as far as the matrices (3.23) and $\tilde{\sigma}_{\alpha \beta}^{0}:=\tilde{\sigma}_{\alpha \beta}^{\underline{b}} u_{\underline{b}}{ }^{0}, \tilde{\sigma}_{\alpha \beta}^{i}:=\tilde{\sigma}_{\alpha \beta}^{\underline{b}} u_{\underline{b}}{ }^{i}$ do possess the algebraic properties of $\mathrm{D}=10$ Pauli matrices. However, one should keep in mind that they are not constant matrices but rather obey

$$
D \sigma_{\alpha \beta}^{0}=\sigma_{\alpha \beta}^{i} \Omega^{i}, \quad D \sigma_{\alpha \beta}^{i}=\sigma_{\alpha \beta}^{0} \Omega^{i},
$$

where $\Omega^{i}$ is defined in (2.12). In this notation the the general solution of Eq.(3.22) reads

$$
h_{\alpha \beta}=\sigma_{\alpha \beta}^{0} .
$$

This is the place to comment on the worldvolume gauge field constraints for the D0-brane case (worldline gauge field). For the $p=0$ the r.h.s. of Eq. (2.14) clearly vanishes so that the constraints read $F_{2}:=d A-\hat{B}_{2}=0$ and the Bianchi identities (2.16) simplify to $\hat{H}_{3}=0$. Their only nontrivial consequence reads

$$
h \tilde{\sigma}^{0} h^{T}=\sigma^{0} .
$$

Eq. (3.26) is satisfied identically by the general solution (3.25) of Eq. (3.22). This shows that the gauge field constraints in the case of D0-brane are dependent, which is in agreement with the known statement that the superembedding equation alone is sufficient to describe dynamics in this case. On the other hand, using both (3.22) and (3.26), one finds the solution (3.25) immediately, much easier than using only (3.22); this illustrates that the use of the superspace constraints for the worldvolume gauge fields (2.14) is helpful also in the cases when the superembedding equation is sufficient to describe the brane dynamics.

Another consequence of the selfconsistency conditions for the superembedding equation (3.20) is that $\Omega^{i}$ in (3.24) is expressed by

$$
\Omega^{i}=e^{0} K^{i}-2 i e^{\beta}\left(\sigma^{0} \tilde{\sigma}^{i} \chi\right)_{\beta}
$$


in terms of fermionic superfield $\chi_{\alpha}=\hat{E}_{0_{\alpha}}^{2}$ and bosonic superfield

$$
K^{i}:=-u_{\underline{a}}^{i} D_{0} \hat{E}_{0}^{\underline{a}}, \quad \hat{E}_{0}^{\underline{a}}:=\nabla_{0} \hat{Z} \underline{M} E_{\underline{M}} \underline{a}(\hat{Z}) .
$$

This latter has a meaning of mean curvatures of the D0-brane (super)worldline in target type IIA superspace. The bosonic and fermionic equations, which can be now obtained from the selfconsistency condition for the fermionic conditions (3.21), are formulated in terms of these superfields. In flat target superspace the equations of motion imply vanishing of both $\chi_{\alpha}$ and $K^{i}$,

$$
\chi_{\alpha}:=\hat{E}_{0 \alpha}^{2}=0, \quad K^{i}:=-u_{\underline{a}}^{i} D_{0} \hat{E}_{0} \underline{a}=0 .
$$

In general type IIA supergravity background the fermionic equations of motion acquires the r.h.s.

$$
\chi_{\alpha}:=\hat{E}_{0 \alpha}^{2}=\Lambda_{\alpha}
$$

defined by

$$
\Lambda_{\alpha}:=\left(\hat{\Lambda}_{1}-\hat{\Lambda}_{2} \sigma^{0}\right)_{\alpha}, \quad \Lambda_{\alpha 1}:=\frac{i}{2}\left(D_{\alpha 1} \Phi\right), \quad \Lambda_{2}^{\alpha}:=\frac{i}{2}\left(D_{2}^{\alpha} \Phi\right),
$$

in terms of pull-backs of the Grassmann derivatives of the dilaton superfield. The origin of this r.h.s. is nonvanishing fermionic torsion of the target type IIA superspace [30]

$$
\begin{gathered}
T^{\alpha 1}=-2 i E^{\alpha 1} \wedge E^{\beta 1} \Lambda_{\beta 1}+i E^{1} \sigma^{\underline{a}} \wedge E^{1} \tilde{\sigma}_{\underline{a}}^{\alpha \beta} \Lambda_{\beta 1}+\propto E^{\underline{b}}, \\
T_{\alpha}^{2}=-2 i E_{\alpha}^{2} \wedge E_{\beta}^{2} \Lambda_{2}^{\beta}+i E^{2} \tilde{\sigma}_{\underline{a}} \wedge E^{2} \sigma_{\alpha \beta}^{\underline{a}} \Lambda_{2}^{\beta}+\propto E^{\underline{b}} .
\end{gathered}
$$

The bosonic equation for D0-brane in general supergravity background reads

$$
\begin{aligned}
& K^{i}:=-u_{\underline{a}}^{i} D_{0} \hat{E}_{0}^{a}=\frac{1}{16} \tilde{\sigma}^{i \alpha \beta}\left(t_{\alpha \beta}-D_{\alpha} \Lambda_{\beta}\right)+\frac{7 i}{8}\left(\hat{\Lambda}_{2} \sigma^{0 i} \hat{\Lambda}_{1}\right)= \\
& =e^{\hat{\Phi}} \hat{R}^{0 i}+\widehat{D^{i} \Phi}+\mathcal{O}\left(\text { fermi } i^{2}\right) \text {, }
\end{aligned}
$$

where $t_{\alpha \beta}=\left(\widehat{T}_{\alpha 1} \underline{a}_{\beta}^{2}+\sigma_{\alpha \gamma}^{0} \widehat{T}_{2}^{\gamma} \underline{a}_{\beta}^{2}-\widehat{T}_{\alpha 1 \underline{a}}{ }^{\delta 1} \sigma_{\delta \beta}^{0}-\sigma_{\alpha \gamma}^{0} \widehat{T}_{2}^{\gamma} \underline{a}^{\delta 1} \sigma_{\delta \beta}^{0}\right) u^{\underline{a} 0}$. To arrive at the second line of Eq. (3.33), written explicitly up to the fermionic contributions, one has to use also the explicit expressions for $D_{\alpha} \Lambda_{\beta}$ and dimension 1 component of fermionic torsions in terms of the background fluxes; these can be extracted from the results of [30] (see also [34]).

\section{Multiple D0-brane equations from superembedding approach.}

It is the usual expectation that the action for a system of $\mathrm{N} \mathrm{D} p$-branes will essentially be a nonlinear generalization of the U(N) SYM action. In particular, the (purely bosonic and not Lorentz invariant) Myers action [20] is of this type. Then the equations of motion which should follow from a hypothetical supersymmetric and Lorentz covariant generalization (or modification) of this action are expected to contain the SU(N) SYM equations $(U(N)=S U(N) \times U(1))$ while the center of mass motion is expected to be described by a usual type of coordinate functions $\hat{Z} \underline{M}(\xi)$ and by related equations for the $\mathrm{U}(1)$ gauge fields (presumably coupled to the $\mathrm{SU}(\mathrm{N})$ equations). Notice that the center of mass equations of motion (and equations for $\mathrm{U}(1)$ gauge fields which is expected to be involved in the center of mass supermultiplet) are expected to be quite close to the equations for a single $\mathrm{D} p$-brane, but with the single brane tension $T$ replaced by $N T$. Our task now is to apply the superembedding approach in search for such supersymmetric equations in the simplest $p=0$ case. 


\subsection{Non-Abelian $\mathcal{N}=16, d=1 \mathrm{SYM}$ constraints on D0-brane}

First, as far as the superembedding description of one brane is based on superembedding equation stating, in its form of (2.4), that the pull-back of the target space bosonic vielbein to the worldvolume superspace $\mathcal{W}^{(1 \mid 16)}$ do not have projections on the fermionic vielbein of $\mathcal{W}^{(1 \mid 16)}$, it is natural to expect that the center of mass motion of the system of multiple D0-brane will also obey the superembedding equations 7 .

As far as the superembedding equation puts the D0-brane model on the mass shell, our superembedding approach to NDp-brane model predicts that the center of mass motion will be described by the equations of motion of single brane with tension $N \cdot T$. (In the case of D0-branes, i.e. Dparticles, $T$ has a meaning of the particle mass). Then, in the light of the above discussion, the only possibility to describe the multiple D0-brane system in the framework of superembedding approach seems to be to consider a non-Abelian $S U(N)$ gauge field supermultiplet on the D0-brane worldvolume superspace $W^{(1 \mid 16)}$. (See [25] for more discussion in the context of searching for hypothetical Q7-branes 33.)

This can be defined by an $s u(n)$ valued non-Abelian gauge potential one form $A=e^{0} A_{0}+e^{\alpha} A_{\alpha}$ with the field strength

$$
G_{2}=d A-A \wedge A=\frac{1}{2} e^{\alpha} \wedge e^{\beta} G_{\alpha \beta}+e^{0} \wedge e^{\beta} G_{\beta 0}
$$

To get a nontrivial consequences for the structure of the field strengths $G_{\alpha \beta}, G_{\beta 0}$ one has to impose constraints. A natural possibility is

$$
G_{\alpha \beta}=i \sigma_{\alpha \beta}^{i} \mathbb{X}^{i}
$$

with some $s u(N)$ valued $S O(9)$ vector superfield $\mathbb{X}^{i}$. The Bianchi identities $D G_{2}=d G_{2}-G_{2} \wedge$ $A+A \wedge G_{2} \equiv 0$ are satisfied if $\mathbb{X}^{i}$ obeys

$$
D_{\alpha} \mathbb{X}^{i}=4 i\left(\sigma^{0} \tilde{\sigma}^{i}\right)_{\alpha}^{\beta} \Psi_{\beta}
$$

and $G_{\alpha 0}=i \Psi_{\alpha}+\frac{i}{2}\left(\sigma^{0 i} \Lambda\right)_{\alpha} \mathbb{X}^{i}$. It is natural to call (4.36) superembedding like equation as it gives a non-Abelian $S U(N)$ generalization of the gauge fixed form of the linearized superembedding equation (2.4) (this reads $D_{\alpha} X^{i}=\propto\left(\sigma^{0} \tilde{\sigma}^{i}\left(\Theta^{2}-\Theta^{1}\right)\right)_{\alpha}$, see [13]).

\subsection{Multiple D0-brane equations of motion from $d=1 \mathcal{N}=16 \mathrm{SYM}$ constraints. Flat target superspace. Relation to $\mathrm{D}=10 \mathrm{SYM}$ and M(atrix) model.}

Let us, for simplicity, consider the case of flat target type IIA superspace, in which, on the mass shell of D0-brane, $\Omega^{i}=0$, so that $\sigma_{\alpha \beta}^{0}$ and $\sigma_{\alpha \beta}^{i}$ are covariantly constants, $D \sigma_{\alpha \beta}^{0}=0=D \sigma_{\alpha \beta}^{i}$. In this case the integrability conditions for Eq. (4.36) $\left(D_{(\beta} D_{\alpha)} \mathbb{X}^{i}=\ldots\right)$ result in

$$
D_{\alpha} \Psi_{\beta}=-\frac{1}{2} \sigma_{\alpha \beta}^{i} D_{0} \mathbb{X}^{i}+\frac{1}{16} \sigma_{\alpha \beta}^{0 i j}\left[\mathbb{X}^{i}, \mathbb{X}^{j}\right]
$$

and the integrability conditions for Eq. (4.37), result in 1d Dirac equation of the form

$$
D_{0} \Psi_{\beta}+\frac{1}{4}\left[\left(\sigma^{0 j} \Psi\right)_{\beta}, \mathbb{X}^{j}\right]=0
$$

\footnotetext{
${ }^{7}$ Of course, this is not a proof. But the universality of the superembedding equation, which is valid for all extended objects studied till now in their maximal worldvolume superspace formulations, and the difficulties one arrives at in any attempt to modify it suggest to believe in its necessity. Finally, if a modification of superembedding equation more appropriate do describe multibrane systems were found, we hope that our present study would be useful in search for such a hypothetical modification.
} 
Applying the Grassmann covariant derivative $D_{\alpha}$ to the fermionic Eq. (4.38), one derives, after some algebra, the following set of equations

$$
\begin{aligned}
D_{0} D_{0} \mathbb{X}^{i}-\frac{1}{32}\left[\left[\mathbb{X}^{i}, \mathbb{X}^{j}\right], \mathbb{X}^{j}\right]+\frac{i}{8}\left\{\Psi_{\alpha}, \Psi_{\beta}\right\} \tilde{\sigma}^{i \alpha \beta}=0 \\
{\left[D_{0} \mathbb{X}^{i}, \mathbb{X}^{i}\right]-4 i\left\{\Psi_{\alpha}, \Psi_{\beta}\right\} \tilde{\sigma}^{0 \alpha \beta}=0 . }
\end{aligned}
$$

Eq. (4.39) is a candidate bosonic equation of motion of multiple D0-brane system. Eq. (4.40) has the meaning of $1 \mathrm{~d}$ Gauss low; this appears in gauge theories as an equation of motion for the time component of gauge potential (which usually plays the rôle of Lagrange multiplier).

The appearance of the counterpart of Gauss low (4.40), characteristic of gauge theory, is not occasional. The point is that our equations appear to be the $D=10$ SYM equations dimensionally reduced to $d=1$. The reason beyod this is that our constraints (4.35) for $d=1, \mathcal{N}=16 \mathrm{SYM}$ multiplet on the flat $d=1 \mathcal{N}=16$ superspace (as appears to be the worldvolume superspace of D0-brane when embedded in flat type IIA superspace) can be obtained as a result of dimensional reduction of $D=10$ supersymmetric gauge theory. Indeed, the $D=10 \mathrm{SYM}$ constraints imply vanishing of spinor-spinor component of the field strength, $\mathbb{F}_{\alpha \beta}:=2 \mathbb{D}_{(\alpha} \mathbb{A}_{\beta)}+\left\{\mathbb{A}_{\alpha}, \mathbb{A}_{\beta}\right\}-2 i \sigma_{\alpha \beta} \mathbb{A}_{\underline{a}}=$ 0 . Assuming independence of fields on the nine spacial coordinate, one finds that spacial components $\mathbb{A}_{i}$ of the ten-dimensional field strength are covariant and can be treated as scalar fields $\mathbb{A}_{i}=1 / 2 \mathbb{X}^{i}$. Then the minimal covariant field strength for $d=1 \mathrm{SYM}$ can be defined as $G_{\alpha \beta}:=2 \mathbb{D}_{(\alpha} \mathbb{A}_{\beta)}+$ $\left\{\mathbb{A}_{\alpha}, \mathbb{A}_{\beta}\right\}-2 i \sigma_{\alpha \beta}^{\underline{0}} \mathbb{A}_{\underline{0}}$ and, due to the original $\mathrm{D}=10 \mathrm{SYM}$ constraints, this is equal to $i \sigma^{i} \mathbb{X}^{i}$, as in Eq. (4.35).

The above observation is important, in particular, because it indicates the relation with Matrix model [8]. Indeed, this is described by the Lagrangian obtained by dimensional reduction of the $D=10$ SYM down to $d=1$ [8]. Actually, the $d=1$ dimensional reduction of the $D=10 \mathrm{SYM}$ was the first model used to describe D0-brane dynamics in [7] even before the complete action for super-Dp-branes where constructed in [10].

To resume, for the multiple D0-brane system in flat target type IIA superspace the worldvolume superspace $\mathcal{W}^{(1 \mid 16)}$ is flat and our superembedding approach results in equations which are equivalent to the ones obtained as a result of dimensional reduction of D=10 SYM and coincide with the Matrix model equations. However, it can also be used to describe the multiple D0-brane system in curved supergravity background, where the way through 10D SYM dimensional reduction is obscure.

\section{Multiple Do-branes in curved type IIA background. Polariza- tion by external fluxes.}

In the case of worldvolume superspace of D0-brane moving in curved target type IIA superspace the calculations become more complex due to the presence of bosonic and fermionic background superfields. For instance, instead of (4.37), one finds

$$
D_{\alpha} \Psi_{\beta}=-\frac{1}{2} \sigma_{\alpha \beta}^{i}+\frac{1}{16} \sigma_{\alpha \beta}^{0 i j}\left[\mathbb{X}^{i}, \mathbb{X}^{j}\right]+\hat{\Lambda}_{1 \epsilon} \Psi_{\delta} \Sigma_{1}{ }^{\epsilon \delta}{ }_{\alpha \beta}+\left(\hat{\Lambda}_{2} \sigma^{0}\right)_{\epsilon} \Psi_{\delta} \Sigma_{2}{ }^{\epsilon \delta}{ }_{\alpha \beta}
$$

with spin-tensors $\Sigma_{1,2}{ }^{\epsilon \delta}{ }_{\alpha \beta}$ possessing the properties $\sigma^{a \underline{b}}{ }_{\delta}^{\alpha} \Sigma_{1,2}{ }^{\epsilon \delta}{ }_{\alpha \beta} \propto \sigma^{a b}{ }^{\epsilon}$ and $D_{\gamma} \Sigma_{1,2}{ }_{\alpha \beta} \propto \Lambda$. We will not need an explicit form of these (we leave this and other details for future publication [34]) as our main interest here will be in the algebraic structure of the bosonic equations of motion 8

${ }^{8}$ The fermionic equations of motion in the presence of fluxes have the structure of

$$
\frac{7}{8}\left(D_{0} \Psi-\frac{1}{4}\left[\mathbb{X}^{i},\left(\sigma^{0 i} \Psi\right)\right]\right)=\left(e^{\hat{\Phi}} \hat{R}^{0 i}+\widehat{D^{i} \Phi}\right)\left(\sigma^{0 i} \Psi\right)-\frac{1}{64} \sigma^{0 k}\left(-\frac{1}{2 !} e^{\hat{\Phi}} \hat{R}_{\underline{b c}} \sigma^{\underline{b c}}-\frac{1}{4 !} e^{\hat{\Phi}} \hat{R}_{\underline{b c d e}} \sigma^{\underline{b c d e}}\right) \sigma^{0 k} \Psi+
$$


Up to the fermionic bilinears proportional to the fermionic background fields these read

$$
\begin{aligned}
D_{0} D_{0} \mathbb{X}^{i}-\frac{1}{32}\left[\left[\mathbb{X}^{i}, \mathbb{X}^{j}\right], \mathbb{X}^{j}\right]+\frac{i}{8}\left\{\Psi_{\alpha}, \Psi_{\beta}\right\} \tilde{\sigma}^{i \alpha \beta}= & D_{0} \mathbb{X}^{j} \mathbb{F}^{j, i}+\frac{1}{16}\left[\mathbb{X}^{j}, \mathbb{X}^{k}\right] \mathbb{G}^{j k, i}+ \\
& +\mathcal{O}\left(\hat{\Lambda}_{1,2} \cdot \Psi\right)+\mathcal{O}\left(\hat{\Lambda}_{1,2} \cdot \hat{\Lambda}_{1,2}\right)
\end{aligned}
$$

The $\mathrm{SO}(9)$ tensors $\mathbb{F}^{j, i}$ and $\mathbb{G}^{j k, i}$ in the r.h.s. of (15.42) are expressed in terms of fluxes by

$$
\begin{array}{r}
\mathbb{F}^{j, i}=q_{0} \widehat{D_{0} \Phi} \delta^{i j}+p_{1} \hat{R}^{i j}+q_{2} \hat{H}^{0 i j} . \\
\mathbb{G}^{j k, i}=p_{0} \delta^{i[j} \widehat{D^{k]} \Phi}+q_{1} \delta^{i[j} \hat{R}^{k] 0}+p_{2} \hat{H}^{i j k}+q_{3} \hat{R}^{0 i j k},
\end{array}
$$

where $q_{0,1,2,3}$ and $p_{0,1,2}$ are constant coefficients characterizing couplings to dilaton as well as to electric and magnetic fields strength of 1-form, 2-form, 3-form gauge fields.

Notice that the center of mass motion is factored out and is described by the single D0-brane equations (3.33),

$$
K^{i}:=D_{0} D_{0} \hat{X}^{i}+\ldots=e^{\hat{\Phi}} \hat{R}^{0 i}+\widehat{D^{i} \Phi}+\mathcal{O}\left(\text { fermi }^{2}\right),
$$

$\left(\hat{X}^{i}:=\hat{Z}^{M} E_{M}^{\underline{a}}(\hat{Z}) u_{\underline{a}}{ }^{i}=\hat{X}^{\underline{a}} u_{\underline{a}}{ }^{i}+\ldots\right)$. Comparing this with Eq. (5.42) we see that the multiple D0branes, as described by this equation, acquire interaction with higher form 'electric' and 'magnetic' fields $\hat{H}^{0 i j}:=H_{\underline{a b c}}(\hat{Z}) u^{\underline{a} 0} u^{\underline{\underline{b}} i} u^{\underline{\underline{c} j}}, H^{i j k}:=H_{\underline{a b c}}(\hat{Z}) u^{\underline{a} i} u^{\underline{b} j} u^{\underline{c} k}, \hat{R}^{0 i j k}:=R_{\underline{a b c d}}(\hat{Z}) u^{\underline{a} 0} u^{\underline{b} \underline{i}} u^{\underline{\underline{c}} j} u^{\underline{\underline{d}} k}$. As one D0-brane does not interact with these background, one may say that the multiple D0-brane system is 'polarized' by the external fluxes such that the interaction with higher brane gauge fields is induced, much in the same way as neutral dielectric is polarized and, due to this polarization, interacts with electric field. This is the famous 'dielectric brane' effect observed by Myers in his purely bosonic nonlinear action [20] which, thus, is observed also for the D0-brane equations which have been obtained from the superembedding approach.

\section{Conclusions and discussion}

In this letter we have reported the results of application of superembedding approach to the search for multiple D0-brane equations. For the case of arbitrary (on-shell) type II supergravity background the dynamical equations obtained from the superembedding approach describe the coupling of multiple D0-branes to the higher NS-NS and RR fluxes $\left(H^{0 i j}, H^{i j k}\right.$ and $\left.R^{0 i j k}\right)$. Thus our equations of motion imply the 'polarization' of multiple D0-brane system under external higher form fluxes which makes them behaving like dipoles of charges characteristic for higher $\mathrm{D} p$-branes. This is the content of the so-called 'dielectric brane effect' [20] characteristic for the (purely bosonic) Myers action [20]. Further study of these equations and of possible restrictions which they might put on the embedding of multiple D0 into a given supergravity background and on their interaction is an interesting problem for future study.

In the case of flat tangent superspace, when the background fluxes vanish, the $d=1, N=16$ worldvolume superspace of D0-brane is flat and the dynamical equations for the relative motion of D0-brane 'constituents', which follows from the superembedding approach, are those of the $\mathrm{D}=10$ $\mathrm{SU}(\mathrm{N}) \mathrm{SYM}$ dimensionally reduced down to $d=1$. They, thus, actually coincide with what had been used for the very low energy description of multiple D0-brane system [7] and with the Matrix model equations [8].

The purely bosonic limit of our equations is clearly simpler than the equations of motion following from the Myers action [20]. In this sense, the suggestion of the superembedding approach is that

$$
+\frac{1}{64} \hat{H}^{0 i j} \sigma^{0 k} \sigma^{i j} \sigma^{0 k} \Psi+D_{0} \mathbb{X}^{i}\left(a_{1} \sigma^{0 i} \hat{\Lambda}_{1}+a_{2} \sigma^{i} \hat{\Lambda}_{2}\right)+\frac{1}{16}\left[\mathbb{X}^{i}, \mathbb{X}^{j}\right]\left(b_{1} \sigma^{i j} \hat{\Lambda}_{1}-b_{2} \sigma^{0 i j} \hat{\Lambda}_{2}\right)+\mathcal{O}\left(\hat{\Lambda}_{1,2} \cdot \hat{\Lambda}_{1,2} \cdot \Psi\right)
$$

with some constants $a_{1,2}$ and $b_{1,2}$. 
this simpler equations, together with the single D0-brane equation describing the center of mass motion, actually give the 'complete' description of the multiple D0-brane system. The advantage of this description is that it is supersymmetric and also Lorentz and diffeomorphism covariant, while the Myers proposal [20] possesses neither of these symmetries expected for a system of coincident Dp-branes. Furthermore, as we have already stressed, our superembedding approach also provides the completely supersymmetric and covariant description of the 'dielectric brane effect'. 9

Another important problem for future study is to understand the relation of our results to the model in [22]. As far as our arguments in support of superembedding equation describing center of mass motion of the multiple D0-brane system cannot be considered as a rigorous proof, if a modification of the superembedding equation resulting in a more complicated interaction of D0constituents did exist, a deeper understanding of the above interrelation might suggest the way to obtain it; in this hypothetical case our present study would provide a basis for such a hypothetical modification 10

Notice that a modified superembedding equation does appear in the boundary fermion approach of [24. However, this happens for the superembedding equation describing the embedding of a worldsheet superspace $\mathcal{W}^{(p+1 \mid 16+2 N)}$ enlarged, to describe the 'classical' counterpart of multiple $\mathrm{D} p$-brane system but not just a single $\mathrm{D} p$-brane, by $2 N$ additional fermionic directions, into the standard type II superspace $\Sigma^{(10 \mid 16+16)}$, and the nonvanishing r.h.s. in the counterpart of (2.5),$\hat{E}^{i}=e^{\dot{\alpha}} \chi_{\dot{\alpha}}{ }^{i}$, happens to be proportional to the new fermionic supervielbein forms $e^{\dot{\alpha}}$ $(\dot{\alpha}=1, \ldots, 2 N)$ corresponding to the new boundary fermion directions of $\mathcal{W}^{(p+1 \mid 16+2 N)}$. This, hence, cannot be used as a suggestion in our case, when the boundary fermions are not used.

This is the place to make a more general comment on the the approach of [24] and to stress that our conclusions are not in contradiction with this work. In the second of the articles [24] a prescription was formulated how to reproduce the Myers action from a specific boundary fermion action. Basically it consists in i) fixing some specific gauge on an additional component of gauge potential related to the boundary fermion directions and, then, ii) replacing Poisson brackets by commutators and boundary fermions by matrices. However, in distinction to the original boundary fermion action, the result of this prescription appears to be, besides purely bosonic, also noncovariant with respect to diffeomorphisms and Lorentz symmetry. A prescription of quantizing the boundary fermion in such a way that to reproduce supersymmetric and Lorentz and diffeomorphism covariant result is not known. In our opinion this problem, noticed already in the original articles [24], might be related with an attempt to quantize only the boundary fermion sector leaving the center of mass degrees of freedom classical. A complete quantization of the dynamical system [24, which provides a fine classical (or 'minus one quantized') description of the dynamical system, should clearly result in an effective action describing, besides D-branes, also supergravity degrees of freedom interacting with them. The question whether it is possible to make a quantization of a part of degrees of freedom and to arrive at a covariant and supersymmetric description of multiple D-brane system in this context is similar to the question of existence of multiple D-brane action (without extra degrees of freedom), which was the motivation of our present study.

To conclude, as we have shown in this letter, the lowest dimensional, $p=0$, multiple $\mathrm{D} p$-brane

\footnotetext{
${ }^{9}$ The Myers action was (and is) motivated by that it is derived from T-duality. But let us stress that the starting point for the corresponding chain of duality transformations is the purely-bosonic D=10 non-Abelian Born-Infeld action based on the symmetric trace prescription [11, and that supersymmetric generalization of these 10D symmetric trace BI action is not known, and its existence can be doubted (see discussion in [25]).

${ }^{10}$ To give an idea of the problems one meets on the way of searching for generalization of our approach which might incorporate nonlinear interactions suggested in [22, let us notice that, although the consideration of [22] uses a purely bosonic worldline, the identification of $\kappa$-symmetry with worldline supersymmetry [26] can be used to identify the corresponding superembedding equation. This appears to be $\hat{E}^{i}=\hat{C}_{1} M^{i}$, where $M^{i}$ is some worldvolume function $\left(\propto \frac{\partial M}{\partial p^{i}}\right.$ in the notation of [22]) and $\hat{C}_{1}$ is the pull-back of the RR 1-superform of the type IIA supergravity ( $\propto d \theta_{1}, \theta_{2}-\theta_{1} d \theta_{2}$ in the case of flat superspace considered in [22]). The problem with such a generalization of superembedding equation is that it is not invariant under the gauge symmetry of the RR 1-form.
} 
system does allow for a description in the frame of superembedding approach. It is interesting to check whether such a description is possible for the case of type IIB D1 (D-string) and type IIA D2-brane (D-membrane). 11 We hope to turn to this problem in future publications.

\section{Acknowledgments}

The author is grateful to Paolo Pasti, Mario Tonin and especially Dmitri Sorokin for useful discussions and comments, as well as for their kind hospitality at the Padova section of INFN and Padova University at early stages of this work which was partially supported by research grants from the Spanish MICINN (FIS2008-1980) and the Ukrainian National Academy of Sciences and Russian Federation RFFI (38/50-2008). The author also thanks Ulf Lindström and Warren Siegel for useful conversations and the organizers of the 2009 Simons Workshop in Mathematics and Physics for their hospitality at Stony Brook at the final stage of this work.

\section{References}

[1] A. Sagnotti, Open strings and their symmetry groups, in: NATO Advanced Summer Institute on Nonperturbative Quantum Field Theory (Cargese Summer Institute) Cargese, France, Jul 16-30, 1987, G. 't Hooft, A. Jaffe, G. Mack, P.K. Mitter, R. Stora Eds., Plenum Press, 1988, pp. 521-528 [ Preptrint ROM2F-87-25 now also available as arXiv:hep-th/0208020.

[2] P. Horava, Strings on world sheet orbifolds, Nucl. Phys. B327, 461 (1989); Background duality of open string models, Phys. Lett. B231, 251 (1989).

[3] J. Dai, R. G. Leigh and J. Polchinski, New Connections Between String Theories, Mod. Phys. Lett. A4, 2073 (1989).

[4] R. G. Leigh, Dirac-Born-Infeld action from Dirichlet sigma model, Mod. Phys. Lett. A 4, 2767 (1989).

[5] J. Polchinski, Dirichlet-Branes and Ramond-Ramond Charges, Phys. Rev. Lett. 75, 4724 (1995) hep-th/9510017.

[6] E. Witten, Bound states of strings and p-branes, Nucl. Phys. B460, 335 (1996) hep-th/9510135.

[7] U. H. Danielsson, G. Ferretti and B. Sundborg, D-particle Dynamics and Bound States, Int. J. Mod. Phys. A11, 5463 (1996) hep-th/9603081]; D. N. Kabat and P. Pouliot, A Comment on Zero-brane Quantum Mechanics, Phys. Rev. Lett. 77, 1004-1007 (1996) hep-th/9603127;

M. R. Douglas, D. N. Kabat, P. Pouliot and S. H. Shenker, D-branes and short distances in string theory, Nucl. Phys. B485, 85-127 (1997) hep-th/9608024.

[8] T. Banks, W. Fischler, S. H. Shenker and L. Susskind, $M$ theory as a matrix model: A conjecture, Phys. Rev. D55, 5112-5128 (1997) hep-th/9610043.

[9] P. K. Townsend, D-branes from M-branes, Phys. Lett. B373, 68-75 (1996) hep-th/9512062.

[10] M. Cederwall, A. von Gussich, B.E.W. Nilsson, A. Westerberg, The Dirichlet super-three-branes in ten-dimensional type IIA and IIB supergravity, Nucl.Phys. B490 (1997) 163-178 hep-th/9610148; M. Cederwall, A. von Gussich, B.E.W. Nilsson, P. Sundell and A. Westerberg, The Dirichlet super-pbranes in ten-dimensional type IIB supergravity, Nucl.Phys. B490 (1997) 179-201 hep-th/9611159.

M. Aganagic, C. Popescu, J.H. Schwarz, D-brane actions with local kappa symmetry, Phys.Lett. B393 (1997) 311-315, hep-th/9610249]; Gauge-invariant and gauge-fixed D-brane actions, Nucl.Phys. B490 (1997) 202, hep-th/9612080;

E. Bergshoeff, P.K. Townsend, Super-D-branes, Nucl.Phys. B490 (1997) 145-162, hep-th/9611173.

\footnotetext{
${ }^{11}$ More generally, it is interesting what is the limiting value $p_{c}$ of the worldspace dimension $p$ above which such a supersymmetric and Lorentz covariant description of multiple of $\mathrm{D} p$-brane dynamics is not possible. The study of [25] suggests that $p_{c}<7$, although a more direct check would not be excessive.
} 
[11] A. A. Tseytlin, Born-Infeld action, supersymmetry and string theory, in The many faces of the superworld. Yuri Golfand memorial volume, M.A. Shifman, M.A. (ed.), World Scientific 2000, pp. 417-452 hep-th/9908105.

[12] M. B. Green, C. M. Hull and P. K. Townsend, D-Brane Wess-Zumino Actions, T-Duality and the Cosmological Constant, Phys. Lett. B382, 65 (1996) hep-th/9604119.

[13] P. S. Howe and E. Sezgin, Superbranes, Phys. Lett. B 390, 133 (1997) hep-th/9607227].

[14] I. A. Bandos, D. P. Sorokin and M. Tonin, Generalized action principle and superfield equations of motion for $D=10$ Dp-branes, Nucl. Phys. B497, 275-296 (1997) hep-th/9701127.

[15] I. Bandos and D. Sorokin, Aspects of D-brane dynamics in supergravity backgrounds with fluxes, kappasymmetry and equations of motion. IIB story, Nucl. Phys. B759, 399 (2006) hep-th/0607163.

[16] I. A. Bandos, D. P. Sorokin, M. Tonin, P. Pasti and D. V. Volkov, Superstrings and supermembranes in the doubly supersymmetric geometrical approach, Nucl. Phys. B446, 79-118 (1995) hep-th/9501113.

[17] P. S. Howe and E. Sezgin, $D=11, p=5$, Phys. Lett. B394, 62 (1997) hep-th/9611008.

[18] I. A. Bandos, K. Lechner, A. Nurmagambetov, P. Pasti, D. P. Sorokin and M. Tonin, Covariant action for the super-five-brane of M-theory, Phys. Rev. Lett. 78 (1997) 4332 hep-th/9701149;

M. Aganagic, J. Park, C. Popescu and J. H. Schwarz, World-volume action of the M-theory five-brane, Nucl. Phys. B496, 191-214 (1997) hep-th/9701166.

[19] A. A. Tseytlin, On non-abelian generalisation of the Born-Infeld action in string theory, Nucl. Phys. B501, 41-52 (1997) hep-th/9701125.

[20] R. C. Myers, Dielectric-branes, JHEP 9912, 022 (1999) hep-th/9910053.

[21] D. P. Sorokin, Coincident (super)-Dp-branes of codimension one, JHEP 08, 022(2001) hep-th/0106212; Space-time symmetries and supersymmetry of coincident D-branes, Fortsch. Phys. 50, 973 (2002);

[22] S. Panda and D. Sorokin, Supersymmetric and kappa-invariant coincident D0-branes, JHEP 0302 (2003) 055 hep-th/0301065.

[23] J. M. Drummond, P. S. Howe and U. Lindstrom, Kappa-symmetric non-Abelian Born-Infeld actions in three dimensions, Class. Quant. Grav. 19, 6477 (2002) hep-th/0206148.

[24] P. S. Howe, U. Lindstrom and L. Wulff, Superstrings with boundary fermions, JHEP 08, 041 (2005) hep-th/0505067; On the covariance of the Dirac-Born-Infeld-Myers action, JHEP 02, 070 (2007) hep-th/0607156; Kappa-symmetry for coincident D-branes, JHEP 09, 010 (2007) [hep-th/0706.2494].

[25] I. A. Bandos, On superembedding approach to type IIB 7-branes, JHEP 0904, 085 (2009) arXiv:0812.2889 [hep-th]].

[26] D. P. Sorokin, V. I. Tkach and D. V. Volkov, Superparticles, twistors and Siegel symmetry, Mod. Phys. Lett. A4 (1989) 901-908.

[27] E. A. Ivanov and A. A. Kapustnikov, Towards a tensor calculus for kappa supersymmetry, Phys. Lett. B 267, 175 (1991); J. P. Gauntlett, A kappa symmetry calculus for superparticles, Phys. Lett. B 272, 25 (1991) arXiv:hep-th/9109039]; A. I. Pashnev and D. P. Sorokin, Note on superfield formulations of $D=2, D=3, D=4, D=6$ and $D=10$ superparticles, Class. Quant. Grav. 10, 625 (1993).

[28] M. Tonin, $\kappa$ symmetry as world sheet supersymmetry in $D=10$ heterotic superstring, Int. J. Mod. Phys. A7, 6013 (1992); F. Delduc, A. Galperin, P. S. Howe and E. Sokatchev, A Twistor formulation of the heterotic $D=10$ superstring with manifest $(8,0)$ world sheet supersymmetry, Phys. Rev. D47, 578 (1993) hep-th/9207050] and refs. in [29].

[29] D. P. Sorokin, Superbranes and superembeddings, Phys. Rept. 329, 1 (2000) hep-th/9906142.

[30] S. Bellucci, S. J. J. Gates, B. Radak and S. Vashakidze, Improved supergeometries for type-II GreenSchwarz non-linear sigma-model, Mod. Phys. Lett. A 4, 1985-1998 (1989).

[31] V. Akulov, I. A. Bandos, W. Kummer and V. Zima, D = 10 Dirichlet super-9-brane, Nucl. Phys. B527, 61 (1998) hep-th/9802032. 
[32] C. S. Chu, P. S. Howe and E. Sezgin, Strings and D-branes with boundaries, Phys. Lett. B428, 59-67 (1998) hep-th/9801202 ; C. S. Chu, P. S. Howe, E. Sezgin and P. C. West, Open superbranes, Phys. Lett. B429, 273-280 (1998) hep-th/9803041.

[33] E. Bergshoeff, J. Hartong and D. Sorokin, Q7-branes and their coupling to IIB supergravity, JHEP 0712, 079 (2007) arXiv:0708.2287 [hep-th]].

[34] Paper in preparation. 